\title{
Extensions of Hénon maps to the closed 4-ball
}

\author{
Gregery T. Buzzard \\ Department of Mathematics, Indiana University, Bloomington, IN 47405
}

\begin{abstract}
Any polynomial automorphism of $\mathbb{C}^{2}$ with nontrivial dynamics is conjugate to a diffeomorphism of the 4-ball such that this diffeomorphism extends to a diffeomorphism of the closed 4-ball. Moreover, the conjugating map is a smooth bijection of $\mathbb{C}^{2}$ to itself. On the sphere at infinity, the extension has an attracting and a repelling solenoid, and the dynamics near these invariant solenoids are described by conjugation to a model solenoidal map.
\end{abstract}

\section{Introduction}

An outgrowth of the great successes in the study of complex dynamics in one variable is the recent attention given to the dynamical behavior of polynomial automorphisms of $\mathbb{C}^{2}$; e.g., by Friedland and Milnor, Bedford and Smillie, Fornæss and Sibony, Hubbard, ObersteVorth, Papadopol, Veselov, and others. In particular, the potential theoretic techniques first developed in one variable have proven to be invaluable in higher dimensions. An introduction to this subject, with further references, can be found in [5]. On the other hand, there is a large body of work on the dynamics of diffeomorphisms of compact manifolds, and some of these techniques have also proven to be of great value in exploring the dynamics of holomorphic maps in several variables. [10] is one introduction to this branch of real dynamics, and [3] and [4] give some applications to holomorphic dynamics. In the hope of providing further links between real dynamics on compact manifolds and polynomial automorphisms of $\mathbb{C}^{2}$, it is natural to search for ways to extend a polynomial automorphism of $\mathbb{C}^{2}$ to a diffeomorphism of a compact manifold. Since there is no obvious way of extending a general polynomial automorphism to a holomorphic (or even real-analytic) diffeomorphism of a compact manifold, we look instead for a smooth extension.

This paper presents one method for constructing such a smooth extension to a compact manifold with boundary: Given a polynomial automorphism with nontrivial dynamics, it is conjugate to a diffeomorphism of the 4-ball such that this diffeomorphism extends to a diffeomorphism of the closed 4-ball. Moreover, the conjugating map is a smooth bijection of $\mathbb{C}^{2}$ to itself. On the sphere at infinity, the extension has an attracting and a repelling solenoid, and the dynamics near these invariant solenoids are described by conjugation to a model solenoidal map introduced by [8]. The methods used here are analytic in character: cut-off functions are used to piece together part of the original automorphism and part of a map which extends naturally to the 3 -sphere at infinity. This piecing together is done in a parametrized way, and the resulting one-parameter family of maps is used to construct an isotopy which forms the basis of the conjugating map.

Some of the results in this paper are similar to portions of the work by Hubbard and Oberste-Vorth [8] and Hubbard, Papadopol, and Veselov [9]. In particular, those papers also describe the extension of polynomial automorphisms to homeomorphisms of the closed 
4-ball. However, the techniques of those papers owe more to algebraic geometry than to analysis. This algebraic-geometric approach has the advantage of preserving as much of the complex analytic structure as possible, but it leads to obstructions which prevent the resulting homeomorphism from being a global diffeomorphism.

For clarity of presentation, we first consider the case of a single Hénon map. In section 2, we construct a homeomorphism of $\mathbb{C}^{2}$ which equals a given Hénon map on a large compact set, which extends to a homeomorphism of the closed 4-ball obtained by adding a 3-sphere at infinity, and which is a diffeomorphism outside of a small set. The extension to the 3-sphere at infinity given by this homeomorphism is interesting in itself and is perhaps the simplest possible extension (see equation (2.1)). This construction is done in a parametrized way to give a one-parameter family containing the original Hénon map. In section 3, we use this oneparameter family together with some cut-off functions to construct an isotopy which maps a fundamental domain for the Hénon map to a fundamental domain for the map constructed in section 2 and which conjugates the two maps on their fundamental domains. This isotopy then extends easily to give a global conjugacy of the Hénon map to the other members of the one-parameter family. In section 4, we show that on the 3-sphere at infinity, the extended map has two invariant solenoids, one attracting and one repelling, and that the dynamics on large parts of this 3-sphere can be described by conjugation to a model solenoidal map. In section 5 we modify the proofs in the previous sections to obtain the same extension and conjugation results, but now using $C^{\infty}$ smooth maps. Finally, in section 6 , we discuss the corresponding results for compositions of several Hénon maps.

I am grateful to Eric Bedford and John Hubbard for several helpful discussions. This research was supported in part by a grant from the National Science Foundation.

\section{A family of homeomorphisms}

In this section we construct a homeomorphism of $\mathbb{C}^{2}$ which equals a given Hénon map on a large bidisk and which extends to the closed 4-ball obtained by adding a 3-sphere at infinity.

First we fix some notation which will be used throughout the paper. Let $F(x, y)=$ $(y, p(y)-a x)$ for $x, y \in \mathbb{C}$, with $a \neq 0$ and $p(y)=y^{d}+\hat{p}(y)$ a polynomial with $d \geq 2$, $\hat{p}(y)=b y^{d-k}+O\left(y^{d-k-1}\right)$ a polynomial, $k \geq 2, b \neq 0$. A map of this form is called a Hénon map and is a polynomial automorphism of $\mathbb{C}^{2}$. By [6], any polynomial automorphism of $\mathbb{C}^{2}$ with nontrivial dynamics is conjugate to a composition of Hénon maps.

Choose $r_{0} \geq \max \{8|a|+8,16|b|\}$ large enough that if $|y| \geq r_{0} / 2$, then $\left|p(y)-y^{d}\right|=$ $|\hat{p}(y)| \leq 2|b||y|^{d-2}$ (hence $\left.\leq|y|^{d-1} / 4\right)$ and $\left|\hat{p}^{\prime}(y)\right| \leq|y|^{d-2}$.

For $r \geq r_{0}$, let

$$
H_{r}(x, y)=\left(y, R \frac{y^{d}}{|y|^{d-1}}-x\right),
$$

where $R=R(r)$ is a smooth positive function to be specified in lemma 2.2, and where we make the standing assumption that $y^{d} /|y|^{d-1}=0$ if $y=0$. Then each $H_{r}$ is a homeomorphism 
of $\mathbb{C}^{2}$ to itself which is real-analytic on $\mathbb{C}^{2}-\{(x, 0): x \in \mathbb{C}\}$. Also, $H_{r}$ is $\mathbb{R}^{+}$homogeneous of degree 1 , hence defines a map of $S^{3}$ to itself. Moreover, $H_{r}$ is conjugate to its inverse by the involution $\sigma(x, y)=(y, x)$. I.e., $H_{r}^{-1}=\sigma^{-1} H_{r} \sigma$.

Notation: Let $S_{r}(y)=R(r) y^{d} /|y|^{d-1}$. Also, let $\|(x, y)\|_{\infty}=\max \{|x|,|y|\}$.

PROPOSITION 2.1 There exists a one-parameter family of homeomorphisms $F_{r}, r \geq r_{0}$ of $\mathbb{C}^{2}$ and a $C^{\infty}$ function $R_{0}(r)>r$ such that $F_{r}(x, y)=F(x, y)$ if $\|(x, y)\|_{\infty}<r$ and $F_{r}(x, y)=H_{r}(x, y)$ if $\|(x, y)\|_{\infty}>R_{0}(r)$. Moreover, $F_{r}(x, y)$ is $C^{\infty}$ in $(r, x, y)$ except on the set $\left\{(r, x, 0): r \geq r_{0},|x| \geq r\right\}$.

Before proving this proposition, we need some preliminaries and a lemma.

Choose a $C^{\infty}$ function $\phi: \mathbb{R} \rightarrow[0,1]$ such that $\phi(t)=1$ if $t \leq 1 / 8, \phi(t)=0$ if $t \geq 7 / 8$, and $-2 \leq \phi^{\prime}(t) \leq 0$. For $y \in \mathbb{C}$, let $\phi_{r}(y)=\phi(|y|-r)$.

Notation: For a smooth function $f: \mathbb{C} \rightarrow \mathbb{C}$, we write $D_{z} f$ for the $2 \times 2$ matrix representing the Jacobian of $f$ at $z$. If $f$ is holomorphic, then $D_{z} f=f^{\prime}(z)$ with the usual identification. For a matrix $A$, we use the notation $\|A\|_{m}$ for the minimum $\min \{\|A v\|:\|v\|=1\}$ and $\|A\|$ for the usual matrix norm. Note that if $f$ is holomorphic, then the matrix norm $\left\|f^{\prime}(z)\right\|$ equals $\left|f^{\prime}(z)\right|$.

LEMMA 2.2 There exists a function $p_{r}: \mathbb{C} \rightarrow \mathbb{C}$ which is $C^{\infty}$ for $(r, y) \in\left[r_{0}, \infty\right) \times \mathbb{C}$ and a $C^{\infty}$ function $R:\left[r_{0}, \infty\right) \rightarrow \mathbb{R}$ with $R=R(r) \geq(r+1)^{d-1}$ such that

1. $p_{r}(y)=p(y)$ if $|y| \leq r$.

2. $p_{r}(y)=S_{r}(y) \quad$ if $|y| \geq r+2$.

3. $\operatorname{Re} \frac{p_{r}(y)}{y^{d}} \geq \frac{r^{d-1}}{2|y|^{d-1}}$ if $|y| \geq r$.

4. $\left\|D_{y} p_{r}\right\|_{m} \geq \begin{cases}\left|p^{\prime}(y)\right| & \text { if }|y| \leq r \\ \frac{d}{2}|y|^{d-1} & \text { if } r \leq|y| \leq r+1 \\ (r+1)^{d-1} & \text { if }|y| \geq r+1\end{cases}$

Proof of Lemma 2.2: For $|y| \leq r+1$, let

$$
p_{r}(y)=y^{d}+\phi_{r}(y) \hat{p}(y)
$$

Then $p_{r}(y)=p(y)$ if $|y| \leq r+1 / 8$, and $p_{r}(y)=y^{d}$ if $r+7 / 8 \leq|y| \leq r+1$. Moreover, if $|y| \geq r_{0} / 2$, then by the choice of $r_{0}$,

$$
\begin{aligned}
\left\|D_{y}\left(\phi_{r}(y) \hat{p}(y)\right)\right\| & \leq\left|\phi_{r}^{\prime}(|y|)\right||\hat{p}(y)|+\phi_{r}(y)\left|\hat{p}^{\prime}(y)\right| \\
& \leq 4|b||y|^{d-2}+|y|^{d-2} .
\end{aligned}
$$


Hence for $r+1 \geq|y| \geq r \geq r_{0}$,

$$
\begin{aligned}
\left\|D_{y} p_{r}\right\|_{m} & \geq\left\|D_{y} y^{d}\right\|_{m}-(4|b|+1)|y|^{d-2} \\
& \geq d|y|^{d-1}-\frac{4|b|+1}{r_{0}}|y|^{d-1} \\
& \geq \frac{d}{2}|y|^{d-1} .
\end{aligned}
$$

Thus condition (1) is satisfied, as are the first two parts of condition (4).

Next we need to pass from $y^{d}$ to $S_{r}(y)=R y^{d} /|y|^{d-1}$. For $R, t \geq 0$, define

$$
A_{r}(t, R)=\int_{0}^{t}\left(d s^{d-1} \phi_{r+1}(s)+\left(1-\phi_{r+1}(s)\right) R\right) d s .
$$

Then $A_{r}(t, R)=t^{d}$ if $t \leq r+9 / 8$ and $\frac{\partial}{\partial t} A_{r}(t, R)=R$ if $t \geq r+2$ We will define $R=$ $R(r)$ implicitly by $A_{r}(r+2, R(r))=R(r)(r+2)$. To see that $R$ can be so defined, let $B(r, R)=A_{r}(r+2, R)-(r+2) R$. Then using the fact that $0 \leq \phi_{r+1}(t) \leq 1$, we see that $(\partial / \partial R) B(r, R)<0$, and integrating first from 0 to $r+1$, then from $r+1$ to $r+2$ shows that $B\left(r,(r+1)^{d-1}\right)>0$ while $B\left(r, d(r+2)^{d-1}\right)<0$.

Hence $R(r)$ is well-defined, is unique, and by the implicit function theorem is $C^{\infty}$. Moreover, $R(r) \geq(r+1)^{d-1}$. For $t \geq 0$, let $A_{r}(t)=A_{r}(t, R(r))$. Then $A_{r}(t)=t^{d}$ for $t \leq r+9 / 8$ and $A_{r}(t)=R(r) t$ for $t \geq r+2$. Also, $A_{r}^{\prime}(t) \geq(r+1)^{d-1}$ if $r+1 \leq t \leq r+2$.

For $|y| \geq r+1$, let

$$
p_{r}(y)=A_{r}(|y|) \frac{y^{d}}{|y|^{d}} .
$$

Then condition (2) is satisfied, and $p_{r}(y)$ is $C^{\infty}$ in $(r, y)$. To check the remaining part of condition (4), we calculate $\left\|D_{y} p_{r}\right\|_{m}$ for $|y| \geq r+1$. By the rotational symmetry of $p_{r}(y)$ for $|y| \geq r+1$, it suffices to find $\left\|D_{t} p_{r}\right\|_{m}$ for $t>r+1$. Note that for $\epsilon$ real and near 0 ,

$$
p_{r}(t+i \epsilon)=\frac{t^{d}+i d t^{d-1} \epsilon}{|t+i \epsilon|^{d}} A_{r}(|t+i \epsilon|)+O\left(\epsilon^{2}\right),
$$

so using the fact that $\left.\left(\frac{\partial}{\partial \epsilon}|t+i \epsilon|\right)\right|_{\epsilon=0}=0$ and $t^{d}=|t|^{d}$, we obtain

$$
D_{t} p_{r}=\left(\begin{array}{cc}
A_{r}^{\prime}(t) & 0 \\
0 & \frac{d}{t} A_{r}(t)
\end{array}\right) .
$$

Since $A_{r}^{\prime}(t) \geq(r+1)^{d-1}$ and $d A_{r}(t) / t \geq d(r+1)^{d-1}$ for $t \geq r+1$, condition (4) follows.

To verify condition (3), note that if $r \leq|y| \leq r+1$, then $\left|p_{r}(y)-y^{d}\right|=\phi_{r}(y)|\hat{p}(y)| \leq$ $|y|^{d-1} / 2$, so dividing both sides by $|y|^{d}$ and taking real parts gives $\operatorname{Re}\left(p_{r}(y) / y^{d}\right) \geq 1-1 / 2|y| \geq$ $r^{d-1} / 2|y|^{d-1}$, where the last inequality follows from the fact that $|y| \geq r \geq r_{0}>1$.

If $|y| \geq r+1$, then integrating from 0 to $r+1$ and $r+1$ to $|y|$ and using $R(r) \geq(r+1)^{d-1}$ gives

$$
\begin{aligned}
A_{r}(|y|) & \geq(r+1)^{d}+(r+1)^{d-1}(|y|-(r+1)) \\
& =|y|(r+1)^{d-1},
\end{aligned}
$$


and hence $\operatorname{Re}\left(p_{r}(y) / y^{d}\right)=A_{r}(|y|) /|y|^{d} \geq r^{d-1} /|y|^{d-1}$. Hence condition (3) holds.

Proof of Proposition 2.1: Let $p_{r}(y)$ be as in the previous lemma. Note that if $|y| \leq r$, then $p_{r}(y)=p(y)$, and if $|y| \geq r+2$, then $p_{r}(y)=S_{r}(y)$. Also, the map $(x, y) \mapsto\left(y, p_{r}(y)-\right.$ $a x)$ is a homeomorphism of $\mathbb{C}^{2}$ for each fixed $r$.

We next modify this homeomorphism by making a diffeomorphism of the plane for each $y$ which equals the map $x \mapsto p_{r}(y)-a x$ if $|y| \geq r+1$ or if $\|(x, y)\|_{\infty} \leq r$, and which equals $x \mapsto S_{r}(y)-a x$ if $|x|$ is sufficiently large (depending on $r$ ).

Define $\alpha_{r}(y)=\left|p_{r}(y)-S_{r}(y)\right|$. Then $\alpha_{r}(y)$ is continuous in $(r, y)$ and uniformly continuous and bounded on $\left[r_{0}, R\right] \times \mathbb{C}$ for any $R>r_{0}$ since $p_{r}(y)=S_{r}(y)$ for $|y| \geq r+2$. Hence, we can choose a positive $C^{\infty}$ function $L(r)$ with $L(r) \geq \sup \left\{\alpha_{r}(y): y \in \mathbb{C}\right\} /|a|$.

Next, define

$$
\psi_{r}(x)=\phi\left(\frac{|x|-r-2}{3 L(r)}\right) .
$$

Then $\psi_{r}(x)=1$ when $|x| \leq r+2$, and $\psi_{r}(x)=0$ if $|x| \geq r+2+3 L(r)$. Also, using the bounds on $\phi_{r}$ and $\phi^{\prime}$, we see that for $x_{1}, x_{2}, y \in \mathbb{C}$ and $t \in \mathbb{R}$,

$$
\left|\frac{\partial}{\partial t} \psi_{r}\left(x_{1}+x_{2} t\right)\right| \leq \frac{2}{3 L(r)}\left|x_{2}\right| \text {. }
$$

Let

$$
q_{r}(x, y)=\psi_{r}(x) p_{r}(y)+\left(1-\psi_{r}(x)\right) S_{r}(y)
$$

and

$$
G_{r}(x, y)=\left(y, q_{r}(x, y)-a x\right)
$$

Note that $G_{r}(x, y)$ is a continuous map of $\mathbb{C}^{2}$ into itself. To show that $G_{r}$ is a homeomorphism, we see from the form of $G_{r}$ that it suffices to show that the function $g_{r, y}(x)=$ $q_{r}(x, y)-a x$ is a homeomorphism of the plane for each fixed $y$. Given any $x_{1}, x_{2} \in \mathbb{C}$, let $g(t)=g_{r, y}\left(x_{1}+\left(x_{2}-x_{1}\right) t\right)$ for $t \in \mathbb{R}$. Then $g$ is $C^{\infty}$, and using (2.3) and the definition of $\alpha_{r}(y)$ we get

$$
\left|g^{\prime}(t)+a\left(x_{1}-x_{2}\right)\right| \leq \frac{2\left|x_{2}-x_{1}\right|}{3 L(r)} \alpha_{r}(y) .
$$

Integrating $g^{\prime}$ from 0 to 1 and using $\alpha_{r}(y) / L(r) \leq|a|$ gives $\left|g_{r, y}\left(x_{2}\right)-g_{r, y}\left(x_{1}\right)\right| \geq\left|x_{2}-x_{1}\right||a| / 3$. Hence $g_{r, y}$ is injective. Moreover, $g_{r, y}$ is continuous, and $g_{r, y}(x)=S_{r}(y)-a x$ for $|x| \geq$ $r+2+3 L(r)$, hence $g_{r, y}(x)$ extends to $\mathbb{C P}^{1}$ by fixing $\infty$. By compactness, this extension is a homeomorphism of $\mathbb{C P}^{1}$, hence $g_{r, y}$ is a homeomorphism of $\mathbb{C}$. As noted, this implies that $G_{r}$ is a homeomorphism of $\mathbb{C}^{2}$.

For the last modification, first note that $q_{r}(x, y)=S_{r}(y)$ if $|x| \geq r+2+3 L(r)$ or $|y| \geq r+2$. Hence for such $(x, y)$ we have $G_{r}(x, y)=\left(y, S_{r}(y)-a x\right)$. Define $M(r)=r+2+3 L(r)$.

We claim that there exist $C^{\infty}$ functions $a(r, x, y)$ for $(r, x, y) \in\left[r_{0}, \infty\right) \times \mathbb{C}^{2}$, and $R_{0}(r)$ for $r \geq r_{0}$, such that 
1. $R_{0}(r) \geq M(r)+1$.

2. $a(r, x, y)=a x$ if $\|(x, y)\|_{\infty} \leq M(r)$.

3. $a(r, x, y)=x$ if $\|(x, y)\|_{\infty} \geq R_{0}(r)$.

4. For fixed $r$ and $y$, and $c>0$, there exists $c^{\prime} \in \mathbb{C}, c^{\prime} \neq 0$ such that $a\left(r, c e^{i \theta}, y\right)=c^{\prime} e^{i \theta}$ for all $\theta \in \mathbb{R}$.

5. For all $(r, x, y),|a(r, x, y)| \leq \max \{|a x|,|x|\}$.

6. For $t>0, \frac{\partial}{\partial t}|a(r, t, y)|>1-\frac{|1-| a||}{1+|a|}$.

7. For any $|x|<|y|,\left\|D_{x} a(r, x, y)\right\|+\left\|D_{y} a(r, x, y)\right\|<7 \max \{|a|, 1\}$.

To construct such an $a(r, x, y)$, let

$$
a_{1}(r, y)=\exp \left(\phi\left(\frac{|y|-M(r)}{N(r)}\right) \log |a|\right)
$$

where $N(r)>M(r)$ is a positive function to be specified later. Note first that $a_{1}(r, y)=|a|$ if $|y| \leq M(r)$, and $a_{1}(r, y)=1$ if $|y| \geq M(r)+N(r)$. Also, $a_{1}(r, y) \leq \max \{|a|, 1\}$, and given $\epsilon_{0}>0$, we can choose $N(r)>0$ sufficiently large to obtain $\left\|D_{y} a_{1}(r, y)\right\|<\epsilon_{0} /(M(r)+1)$. For $t>0$ define

$$
a_{2}(r, t, y)=t+\int_{0}^{t} \phi_{M(r)}(s)\left(a_{1}(r, y)-1\right) d s .
$$

Then $a_{2}(r, t, y)=a_{1}(r, y) t$ for $t \leq M(r)$, and $a_{2}(r, t, y)-t$ is constant for $t \geq M(r)+1$.

Let $\epsilon(r, t, y)=a_{2}(r, t, y)-t$. Then $\epsilon(r, t, y)=\epsilon(r, M(r)+1, y)$ whenever $t \geq M(r)+1$, and for fixed $r$,

$$
|\epsilon(r, M(r)+1, y)| \leq|1-| a||(M(r)+1) .
$$

Let $\epsilon_{r}=|1-| a||(M(r)+1)$, let $R_{0}(r)=(1+2 N(r)(1+|a|))(M(r)+1)$, and let

$$
a_{3}(r, t, y)=a_{2}(r, t, y)-\left(1-\phi\left(\frac{t-M(r)-1}{R_{0}(r)-M(r)-1}\right)\right) \epsilon(r, M(r)+1, y) .
$$

Then $a_{3}(r, t, y)=a_{2}(r, t, y)$ for $t \leq M(r)+1$, and $a_{3}(r, t, y)=t$ for $t \geq R_{0}(r)$. Moreover, using $\left|\phi^{\prime}\right|<2$, we see that for $t \geq M(r)+1$,

$$
\left|1-\frac{\partial}{\partial t} a_{3}(r, t, y)\right| \leq 2 \frac{\epsilon_{r}}{R_{0}(r)-M(r)-1} \leq \frac{|1-| a||}{N(r)(1+|a|)}
$$

Finally, let

$$
a(r, x, y)=a_{3}(r,|x|, y) \exp \left(i \phi\left(\frac{|x|-M(r)}{N(r)}\right) \phi\left(\frac{|y|-M(r)}{N(r)}\right) \operatorname{Im}(\log a)\right) \frac{x}{|x|},
$$

where $\log a$ is any branch of the $\operatorname{logarithm}$. Then $a(r, x, y)$ satisfies properties 1 through 6 immediately. 
Moreover, for $t>0$, we see from (2.4) and $\left|\phi^{\prime}\right|<2$ that

$$
\left|\frac{\partial}{\partial t} a(r, t, y)\right| \leq \max \left\{|a|, 1+\frac{1}{N(r)}\right\}+\left|a_{3}(r, M(r)+N(r), y)\right| \frac{2}{N(r)},
$$

which by property 5 is bounded by $\left(3+\epsilon_{0}\right) \max \{|a|, 1\}$ for $N(r)$ large. Also, for $t>0$,

$$
\left|\frac{\partial}{\partial \epsilon} a(r, t+i \epsilon, y)\right|_{\epsilon=0}|=| a_{3}(r, t, y) \mid \frac{1}{t},
$$

which again by property 5 is bounded by $\max \{|a|, 1\}$. By rotational symmetry, we see that $\left\|D_{x} a(r, x, y)\right\| \leq\left(4+\epsilon_{0}\right) \max \{|a|, 1\}$.

Next, note that $\left\|D_{y} a_{2}(r,|x|, y)\right\| \leq(M(r)+1)\left\|D_{y} a_{1}(r, y)\right\| \leq \epsilon_{0}$, hence $\left\|D_{y} a_{3}(r,|x|, y)\right\| \leq$ $2 \epsilon_{0}$. Thus

$$
\left\|D_{y} a(r, x, y)\right\| \leq\left\|D_{y} a_{3}(r,|x|, y)\right\|+\left|a_{3}(r,|x|, y)\right|\left\|D_{y} \phi\left(\frac{|y|-M(r)}{N(r)}\right)\right\|,
$$

and since this derivative of $\phi$ is 0 for $|y| \geq M(r)+N(r)$, property 5 implies that if $|x|<|y|$, then the right hand side is bounded by $2 \epsilon_{0}+2(M(r)+N(r)) / N(r)$. Combining this with the bound for $\left\|D_{x} a\right\|$, we see that for $\epsilon_{0}<1 / 4$ and $N(r)$ sufficiently large, property 7 is satisfied also. Thus, $a(r, x, y)$ exists as claimed.

In particular, for each fixed $r$ and $y$, the map $x \mapsto S_{r}(y)-a(r, x, y)$ is a smooth injective map of $\mathbb{C}$ to itself which equals $x \mapsto S_{r}(y)-x$ for $x \geq R_{0}(r)$, and hence this map is a diffeomorphism of $\mathbb{C}$.

Finally, let

$$
F_{r}(x, y)= \begin{cases}G_{r}(x, y)=\left(y, q_{r}(x, y)-a x\right) & \text { if }\|(x, y)\|_{\infty} \leq M(r) \\ \left(y, S_{r}(y)-a(r, x, y)\right) & \text { if }\|(x, y)\|_{\infty} \geq M(r) .\end{cases}
$$

Note that the two definitions agree in a neighborhood of $\left\{\|(x, y)\|_{\infty}=M(r)\right\}$. To show that $F_{r}$ is a homeomorphism of $\mathbb{C}^{2}$ to itself, it suffices to check that for each fixed $y, F_{r}(\cdot, y)$ is a homeomorphism of $\mathbb{C}$ to itself, which follows from the remarks about $S_{r}(y)-a(r, x, y)$.

\section{Conjugation}

In this section we show that the map $F_{r}$ constructed in the previous section is conjugate to the Hénon map $F$. First we develop some of the basic properties of the maps $F_{r}$, then we use the family of maps $F_{r}$ to define an isotopy which can be used to conjugate $F$ to $F_{r}$.

Notation: Let $F_{\infty}=F$.

LEMMA 3.1 Let $r \in\left[r_{0}, \infty\right]$. If $|y| \geq r_{0} / 2$ and $|x| \leq 2|y|$, then $(z, w)=F_{r}(x, y)$ satisfies $|w| \geq 3|z| \geq 3 r_{0} / 2$. 
Proof: Since $F_{\infty}=F_{r}$ on $\Delta^{2}(0 ; r)$ for any $r \geq r_{0}$, we may assume without loss that $r<\infty$. First suppose $\|(x, y)\|_{\infty} \leq M(r)$. Choose $\theta \in \mathbb{R}$ such that $e^{i \theta} y^{d}=|y|^{d}$. Since $|y| \geq r_{0} / 2$, we have $\left|e^{i \theta} p(y)-e^{i \theta} y^{d}\right| \leq|y|^{d} / 4$ by choice of $r_{0}$. If $|y| \leq r$, then $\operatorname{Re}\left(e^{i \theta} p_{r}(y)\right)=$ $\operatorname{Re}\left(e^{i \theta} p(y)\right) \geq 3|y|^{d} / 4$. If $|y| \geq r$, then $\operatorname{Re}\left(e^{i \theta} p_{r}(y)\right)=\operatorname{Re}\left(p_{r}(y) / y^{d}\right)|y|^{d} \geq\left(r^{d-1} / 2\right)|y|$. In either case, $\operatorname{Re}\left(e^{i \theta} p_{r}(y)\right) \geq 3 r_{0}|y| / 8$. Hence

$$
\begin{aligned}
\left|q_{r}(x, y)\right| & \geq \operatorname{Re}\left(e^{i \theta} q_{r}(x, y)\right) \\
& \geq \psi_{r}(x, y) r_{0} \frac{3|y|}{8}+\left(1-\psi_{r}(x, y)\right) R(r)|y| \\
& \geq \frac{3 r_{0}|y|}{8}
\end{aligned}
$$

Since $r_{0} \geq 8|a|+8$ and $|x| \leq 2|y|$, we obtain $|w|=\left|q_{r}(x, y)-a x\right| \geq 3 r_{0}|y| / 8-2|a||y| \geq$ $3|y|=3|z|$. Hence $(z, w)=F_{r}(x, y)$ satisfies $|w| \geq 3|z| \geq 3 r_{0} / 2$.

To finish the proof, suppose $\|(x, y)\|_{\infty} \geq M(r)$, in which case $(x, y)=\left(y, S_{r}(y)-\right.$ $a(r, x, y))$. Then $|w|=\left|S_{r}(y)-a(r, x, y)\right| \geq r_{0}|y|-\max \{|a x|,|x|\} \geq 3|y|=3|z|$. Hence the lemma holds.

LEMMA 3.2 Let $r \in\left[r_{0}, \infty\right]$. If $|x| \geq r_{0} / 2$ and $|y| \leq 2|x|$, then $(z, w)=F_{r}^{-1}(x, y)$ satisfies $|z| \geq 3|w| \geq 3 r_{0} / 2$.

Proof: Again we may assume $r<\infty$. Suppose that $\|(z, w)\|_{\infty} \leq M(r)$. Then $(x, y)=$ $F_{r}(z, w)=\left(w, q_{r}(z, w)-a z\right)$. Since $|w|=|x| \geq r_{0} / 2$, we have as in the previous lemma that $\left|q_{r}(z, w)\right| \geq 3 r_{0}|w| / 8$. Since $|y| \leq 2|w|$, we have $|a z| \geq\left|q_{r}(z, w)\right|-|y| \geq 3 r_{0}|w| / 8-2|w| \geq$ $3|a||w|$. Hence $|z| \geq 3|w| \geq 3 r_{0} / 2$.

If $\|(z, w)\|_{\infty} \geq M(r)$, then $(x, y)=\left(w, S_{r}(w)-a(r, z, w)\right)$, so examining the second coordinate and using property 5 of $a(r, x, y)$ we obtain

$$
\begin{aligned}
\max \{|a z|,|z|\} & \geq|a(r, z, w)| \\
& \geq R(r)|w|-|y| \\
& \geq\left((r+1)^{d-1}-2\right)|w| \\
& \geq(8|a|+6)|w| .
\end{aligned}
$$

Hence $|z| \geq 3|w| \geq 3 r_{0} / 2$, as desired.

Notation: Let $\Delta_{0}^{2}=\left\{(x, y):|x| \leq r_{0} / 2,|y| \leq r_{0}\right\}$.

Notation: For a map $f$, let $\Omega(f)$ denote the nonwandering set of $f$. I.e., $\Omega(f)$ consists of all points $p$ such that for any neighborhood $V$ of $p$, there exists $n>0$ so that $V \cap f^{n}(V) \neq \emptyset$.

LEMMA 3.3 For each $r \geq r_{0}, \Omega\left(F_{r}\right)=\Omega(F)$.

Proof: By [2], $\Omega(F) \subseteq \Delta_{0}^{2}$. By the two previous lemmas, if $\|(x, y)\|_{\infty} \geq r_{0} / 2$, then $(x, y)$ is wandering for $F_{r}$. Since $F=F_{r}$ on $\Delta_{0}^{2}$, the lemma follows. 
Notation: Let $V=\left\{|x|>r_{0} / 2,|x|>|y| / 2\right\}$, and for $r \in\left[r_{0}, \infty\right]$, let $D_{r}=\left(F_{r}(\bar{V}) \backslash V\right) \cup \overline{\Delta_{0}^{2}}$.

LEMMA 3.4 Let $r \in\left[r_{0}, \infty\right]$ and $(x, y) \in \mathbb{C}^{2}$. Then there exists $n \in \mathbb{Z}$ such that $F_{r}^{n}(x, y) \in$ $D_{r}$

Proof: If there exists $n$ so that $F_{r}^{n}(x, y) \in \overline{\Delta_{0}^{2}}$, then the proof is done. Otherwise, lemma 3.2 implies that $\cap_{n \leq 0} F_{r}^{n}(V)=\emptyset$, so $\cup_{n \leq 0} F_{r}^{n}\left(\mathbb{C}^{2} \backslash V\right)=\mathbb{C}^{2}$. Hence there exists $n \geq 0$ such that $F_{r}^{n}(x, y) \in \mathbb{C}^{2} \backslash V$. Similarly, lemma 3.1 implies that there exists $n \leq 0$ so that $F_{r}^{n}(x, y) \in V$. Since $F_{r}^{-1}(V) \subseteq V$ and $F_{r}\left(\mathbb{C}^{2} \backslash V\right) \subseteq \mathbb{C}^{2} \backslash V$, there exists a unique integer $n$ such that $F_{r}^{n-1}(x, y) \in \bar{V}$ and $F_{r}^{n}(x, y) \in \mathbb{C}^{2} \backslash V$, hence $F_{r}^{n}(x, y) \in F_{r}(\bar{V}) \backslash V$ as desired.

Notation: Let $U_{0}=\left\{|x|<r_{0},|y| \leq r_{0}\right\}, U_{1}=\left\{|y| \geq r_{0},|x|<|y|\right\}$, and $U=U_{0} \cup U_{1}$. Note that $U$ is a neighborhood of $\partial V$.

LEMMA 3.5 There exists a neighborhood $V_{0}$ of $\bar{V}$ such that $F_{r}(\bar{U}) \cap \bar{V}_{0}=\emptyset$ for all $r \in$ $\left[r_{0}, \infty\right]$.

Proof: Lemma 3.2 implies that $F^{-1}(\bar{V}) \cap \bar{U}_{0}=\emptyset$, so applying $F$ gives $F\left(\bar{U}_{0}\right) \cap \bar{V}=\emptyset$. Note also that $F_{r}\left(\bar{U}_{0}\right)=F\left(\bar{U}_{0}\right)$ for all $r \geq r_{0}$. Moreover, if $(x, y) \in \bar{U}_{1}$, then by lemma 3.1, $(z, w)=F_{r}(x, y)$ has $|w| \geq 3|z| \geq 3 r_{0} / 2$, which is bounded away from $\bar{V}$. Hence for all $r \in\left[r_{0}, \infty\right], F_{r}(\bar{U})$ is contained in the closed set $F\left(\bar{U}_{0}\right) \cup\left\{(x, y):|y| \geq 3|x| \geq 3 r_{0} / 2\right\}$, and this union does not intersect $\bar{V}$. Hence there exists $V_{0}$ as claimed.

Notation: For $c \in \mathbb{C}$, let $X_{c}=\left\{(c y, y):|y|>r_{0} / 2\right\}$. For $w \in \mathbb{C}$, let $Y_{w}=\{(z, w): z \in \mathbb{C}\}$.

LEMMA 3.6 Let $|c|<1$, and $w \in \mathbb{C}$. Then for all $r \in\left[r_{0}, \infty\right], F_{r}\left(X_{c}\right)$ and $Y_{w}$ are transverse. In particular, given $\left(z_{0}, w_{0}\right) \in F_{r}\left(X_{c}\right) \cap Y_{w_{0}}$, there exists a neighborhood $W$ of $r$ in $\left[r_{0}, \infty\right]$ and a unique $C^{\infty}$ function $z: W \rightarrow \mathbb{C}$ such that $z(r)=z_{0}$ and for all $s \in W$, $\left(z(s), w_{0}\right) \in F_{s}\left(X_{c}\right) \cap Y_{w_{0}}$.

Proof: Let $(z, w) \in F_{r}\left(X_{c}\right) \cap Y_{w}$, where $|c|<1$ and $r \in\left[r_{0}, \infty\right]$. By lemma 3.1, $|w| \geq$ $3 r_{0} / 2$. Moreover, the tangent space of $Y_{w}$ at $(z, w)$ is $\mathbb{C}(1,0)$. Hence to check transversality, it suffices to check that

$$
\left\|D_{y}\left(\pi_{2} F_{r}(c y, y)\right)\right\|_{m}>0 .
$$

In fact, we will prove the stronger result, which will be useful later, that if $|x|<|y|,|y| \geq$ $r_{0} / 2$, and $|c|<1$, then for each real $\theta$,

$$
\left|\left(D_{(x, y)} \pi_{2} F_{r}\right)\left(\begin{array}{c}
c \\
e^{i \theta}
\end{array}\right)\right|>\left|\left(D_{(x, y)} \pi_{1} F_{r}\right)\left(\begin{array}{c}
c \\
e^{i \theta}
\end{array}\right)\right|,
$$

where $D_{(x, y)} \pi_{j} F_{r}$ represents the $2 \times 4$ matrix of partial derivatives of $\pi_{j} F_{r}$ at the point $(x, y)$.

From the definition of $F_{r}$ in equation (2.5), we see that for any $x$ and $y$,

$$
\left(D_{(x, y)} \pi_{1} F_{r}\right)\left(\begin{array}{c}
c \\
e^{i \theta}
\end{array}\right)=e^{i \theta},
$$


and hence in (3.1) it suffices to show that the left hand side is bigger than 1 . The definition of $F_{r}$ also implies that if $\|(x, y)\|_{\infty} \leq M(r)$, then

$$
\pi_{2} F_{r}(x, y)=\psi_{r}(x) p_{r}(y)+\left(1-\psi_{r}(x)\right) S_{r}(y)-a x .
$$

If also $|y| \geq r+2$, then $p_{r}(y)=S_{r}(y)$, so

$$
\pi_{2} F_{r}(x, y)=R(r)|y| \frac{y^{d}}{|y|^{d}}-a x,
$$

and a calculation like that leading to (2.2) together with $R(r)>8|a|+8$ and $|c|<1$ shows that the left hand side of (3.1) is at least $R(r)-|a c|>1$ in this case.

If $|y|<r+2$, then $|x|<r+2$ by assumption, so $\psi_{r}(x)=1$ and hence $\pi_{2} F_{r}(x, y)=$ $p_{r}(y)-a x$. Since $|y|>r_{0} / 2$, part 4 of lemma 2.2 and the choice of $r_{0}$ shows that

$$
\left\|D_{y} p_{r}(y)\right\|_{m} \geq(|y|-1)^{d-1}>4|a|+3
$$

so the left hand side of (3.1) is at least $3|a|+3>1$. Hence (3.1) holds whenever $|x|<|y|$, $|c|<1$, and $r_{0} / 2<|y| \leq M(r)$.

If $|y| \geq M(r)$, then

$$
\pi_{2} F_{r}(c y, y)=R(r)|y| \frac{y^{d}}{|y|^{d}}-a(r, x, y) .
$$

By property 7 of $a(r, x, y)$ and a calculation like that for equation (2.2), we see that the left hand side of (3.1) is at least

$$
R(r)-\left\|D_{x} a(r, x, y)\right\||c|-\left\|D_{y} a(r, x, y)\right\|>1
$$

Hence the transversality part of the current lemma is seen to be true, and the rest of the lemma follows from the implicit function theorem.

LEMMA 3.7 There exists a $C^{\infty}$ map $g:\left[r_{0}, \infty\right] \times \mathbb{C}^{2} \rightarrow \mathbb{C}^{2}$ such that $g_{r}=g(r, \cdot)$ satisfies

1. $g_{\infty}=I d$.

2. $g_{r}$ is a diffeomorphism of $\mathbb{C}^{2}$ and a diffeomorphism of $D_{\infty}$ onto $D_{r}$.

3. $\pi_{2} g_{r}(x, y)=y$ for all $(x, y) \in \mathbb{C}^{2}$ and all $r \in\left[r_{0}, \infty\right]$.

4. $g_{r}=I d$ on a neighborhood (independent of $r$ ) of $\partial V \cup \Delta_{0}^{2}$.

5. $g_{r}\left(F\left(X_{c}\right)\right)=F_{r}\left(X_{c}\right)$ for all $r \in\left[R_{0}, \infty\right]$ and all $|c| \leq 3 / 4$.

Proof: We will define $g_{r}$ as the time- $t$ map $\left(t=r_{0} / r\right)$ of a time dependent vector field which preserves the sets $Y_{w}$ and which is compactly supported within each $Y_{w}$. This approach avoids questions of the completeness of vector fields defined on noncompact sets. First we define an isotopy, $h_{r}$, of $F(U)$ to $F_{r}(U)$ which preserves the sets $Y_{w}$, then use the ideas of the isotopy extension theorem to obtain $g_{r}$. 
Fix $\left(x_{0}, y_{0}\right) \in U$. If $\left|y_{0}\right| \leq r_{0}$, then also $\left|x_{0}\right| \leq r_{0}$, so $F_{r}\left(x_{0}, y_{0}\right)=F\left(x_{0}, y_{0}\right)$ for all $r \in\left[r_{0}, \infty\right]$. Define $h_{r}\left(F\left(x_{0}, y_{0}\right)\right)=F\left(x_{0}, y_{0}\right)$ for all $r \in\left[r_{0}, \infty\right]$.

Next, let $\left(x_{0}, y_{0}\right) \in U$ with $\left|y_{0}\right|>r_{0}$, and let $|c|<1$ such that $x_{0}=c y_{0}$. Since $F_{r}\left(x_{0}, y_{0}\right)=$ $F\left(x_{0}, y_{0}\right)$ for $r \geq\left\|\left(x_{0}, y_{0}\right)\right\|_{\infty}$, we define $h_{r}\left(F\left(x_{0}, y_{0}\right)\right)=F\left(x_{0}, y_{0}\right)$ for such $r$. To complete the definition of $h_{r}$, let $w=\pi_{2} F\left(x_{0}, y_{0}\right)$, and suppose that $h_{r}\left(F\left(x_{0}, y_{0}\right)\right)$ is defined and $C^{\infty}$ for $r \in\left(r_{1}, \infty\right]$ with $h_{r}\left(F\left(x_{0}, y_{0}\right)\right) \in F_{r}\left(X_{c}\right) \cap Y_{w}$ for all $r \in\left(r_{1}, \infty\right]$.

In order to extend $h_{r}\left(F\left(x_{0}, y_{0}\right)\right)$ to $\left[r_{0}, \infty\right]$, we first extend the definition to $r=r_{1}$, then use transversality to extend to a neighborhood of $r_{1}$. By compactness, this is sufficient to extend to all of $\left[r_{0}, \infty\right]$.

The definition of $F_{r}$ shows that $\pi_{2} F_{r}(c y, y)$ behaves like a polynomial in the sense that $\pi_{2} F_{r}(c y, y)=w$ has exactly $d$ zeros counted with multiplicity (note that lemma 3.6 implies that the multiplicity of each zero is 1 except possibly at points where $F_{r}(c y, y)=F(c y, y)$ is holomorphic). In particular, $h_{r_{1}}\left(F\left(x_{0}, y_{0}\right)\right)=\lim _{r \rightarrow r_{1}} h_{r}\left(F\left(x_{0}, y_{0}\right)\right)$ must exist and equal one of the images of the $d$ zeros corresponding to $r=r_{1}$. Simple estimates imply that $h_{r_{1}}\left(F\left(x_{0}, y_{0}\right)\right)$ is contained in $F_{r_{1}}\left(X_{c}\right) \cap Y_{w}$, so lemma 3.6 implies that $h_{r}\left(F\left(x_{0}, y_{0}\right)\right)$ extends uniquely to a neighborhood of $r_{1}$ with $h_{r}\left(F\left(x_{0}, y_{0}\right)\right) \in F_{r}\left(X_{c}\right) \cap Y_{w}$, hence $h_{r}$ extends to $r \in\left[r_{0}, \infty\right]$, as desired.

The implicit function theorem implies that $h_{r}$ is smooth on $\left[r_{0}, \infty\right] \times F(U)$. Note also that $h_{r}=I d$ on $\Delta_{0}^{2}$ for all $r$. As in the isotopy extension theorem, e.g., [7, theorem 8.1.4], the trajectory of a point under $h_{r}$ defines a smooth vector field by

$$
Q_{t}(p)=\left\{\begin{array}{cl}
\left.\left(\frac{d}{d r} h_{r}\left(h_{r_{0} / t}^{-1}(p)\right)\right)\right|_{r=r_{0} / t} & \text { if } t \neq 0 \\
0 & \text { if } t=0
\end{array}\right.
$$

where $p \in h_{r_{0} / t}(F(U))=F_{r_{0} / t}(U)$ and $t \in[0,1]$. Note that $\pi_{2} Q_{t} \equiv 0$, so that $Q_{t}$ restricts to a vector field on each $Y_{w}$.

Let $\mu: \mathbb{C}^{2} \rightarrow[0,1]$ be a $C^{\infty}$ function which is 0 on $\mathbb{C}^{2} \backslash U$ and 1 in a neighborhood of $\mathbb{C}^{2} \backslash V$ and such that $\mu=1$ on each set $X_{c}$ with $|c| \leq 3 / 4$. Then $Z_{t}(p)=\mu\left(F_{r_{0} / t}^{-1}(p)\right) Q_{t}(p)$ is a $C^{\infty}$ vector field for $p \in F_{r_{0} / t}(U), t \in[0,1]$, with $\pi_{2} Z_{t} \equiv 0, Z_{t} \equiv Q_{t}$ on $F_{r_{0} / t}\left(\mathbb{C}^{2} \backslash V\right)$ and on $F_{r_{0} / t}\left(X_{c}\right)$ for each $|c| \leq 3 / 4$, and $Z_{t} \equiv 0$ on a neighborhood of $F_{r_{0} / t}(\partial U)$ and on $\Delta_{0}^{2} \cap F_{r_{0} / t}(U)$ since $h_{r} \equiv I d$ there for any $r$. Hence $Z_{t}$ extends to a smooth vector field on $\mathbb{C}^{2}$ by setting $Z_{t} \equiv 0$ on the complement of $F_{r_{0} / t}(U)$. In particular, $Z_{t}$ preserves each $Y_{w}$, and $Z_{t} \equiv 0$ on $\bar{V}$ by lemma 3.5 , so $Z_{t}$ is complete.

For $r \in\left[r_{0}, \infty\right]$, let $g_{r}$ be the time- $\left(r_{0} / r\right)$ map of $Z_{t}$. Then properties $1-4$ are immediate, and property 5 is true since $h_{r}\left(F\left(X_{c}\right)\right)=F_{r}\left(X_{c}\right)$ and $g_{r}=h_{r}$ on $F\left(X_{c}\right)$ for $|c| \leq 3 / 4$.

THEOREM 3.8 For each $r \geq r_{0}$, the maps $F_{r}$ and $F$ are conjugate via a homeomorphism of $\mathbb{C}^{2}$. I.e., there exists $\Phi_{r}: \mathbb{C}^{2} \rightarrow \mathbb{C}^{2}$ a homeomorphism such that $\Phi_{r} F \Phi_{r}^{-1}=F_{r}$.

Proof: We will construct a homeomorphism $\Phi_{r}: D_{\infty} \rightarrow D_{r}$ such that $\Phi_{r}$ is the identity on $\partial V \cup \overline{\Delta_{0}^{2}}$, and $\Phi_{r}=F_{r} \circ F^{-1}$ on $F(\partial V)$.

Before constructing $\Phi_{r}$, we show how this proves the theorem. Suppose $p \in D_{\infty}$ and $F(p) \in D_{\infty}$. By lemma 3.1, either $p \in \partial V$ or $p \in \overline{\Delta_{0}^{2}}$. If $p \in \partial V$, then $\Phi_{r}(p)=p$, so $F_{r} \Phi_{r}(p)=F_{r}(p)$. Also, $F(p) \in F(\partial V)$, so $\Phi_{r} F(p)=\left(F_{r} F^{-1}\right) F(p)=F_{r}(p)=F_{r} \Phi_{r}(p)$. On 
the other hand, if $p \in \overline{\Delta_{0}^{2}}$ (and $\left.F(p) \in D_{\infty}\right)$, then $F(p) \in \overline{\Delta_{0}^{2}}$, so $\Phi_{r}(p)=p, \Phi_{r} F(p)=F(p)$, and $F(p)=F_{r}(p)$, so $F_{r} \Phi_{r}(p)=\Phi_{r} F(p)$. Hence $F_{r} \Phi_{r}=\Phi_{r} F$ on $D_{\infty} \cap F^{-1}\left(D_{\infty}\right)$.

Moreover, given $\Phi_{r}$ defined on $D_{\infty}$, we can extend $\Phi_{r}$ to $\mathbb{C}^{2}$ by iteration. For $p \in \mathbb{C}^{2}$, lemma 3.4 implies that there exists $n \in \mathbb{Z}$ so that $F^{n}(p) \in D_{\infty}$. Define $\Phi_{r}(p)=F_{r}^{-n} \Phi_{r} F^{n}(p)$. Since $\Phi_{r}(p)=F_{r} \Phi_{r} F(p)$ for $p \in D_{\infty} \cap F^{-1}\left(D_{\infty}\right)$ and $F_{r}=F$ on $\overline{\Delta_{0}^{2}}$, we see that $\Phi_{r}$ is welldefined and continuous. Moreover, $\Phi_{r}^{-1}$ is defined by $\Phi_{r}^{-1}(p)=F^{n} \Phi_{r}^{-1} F_{r}^{n}(p)$, where $n \in \mathbb{Z}$ is chosen so that $F_{r}^{n}(p) \in D_{r}$, and as before, $\Phi_{r}^{-1}$ is well-defined and continuous. Hence $\Phi_{r}$ is a homeomorphism of $\mathbb{C}^{2}$ with $\Phi_{r} F \Phi_{r}^{-1}=F_{r}$ on $\mathbb{C}^{2}$.

To construct $\Phi_{r}$, let $\mu: \mathbb{C}^{2} \rightarrow[0,1]$ be $C^{\infty}$ with $\mu \equiv 0$ in a neighborhood of $F(\partial V) \backslash F\left(\overline{\Delta_{0}^{2}}\right)$ and $\mu \equiv 1$ in a neighborhood of $\partial V \cup \overline{\Delta_{0}^{2}}$. We also require that $\mu$ is constant on each set $F\left(X_{c}\right)$ for $|c| \leq 1 / 2$ and $\mu(p)=1$ for each $p=F(c y, y)$ with $|c| \geq 1 / 2$. Define $\Phi_{r}: D_{\infty} \rightarrow \mathbb{C}^{2}$ by

$$
\Phi_{r}(p)=F_{r} F_{r / \mu(p)}^{-1} g_{r / \mu(p)}(p) .
$$

Note that if $|c| \leq 3 / 4$, then part 5 of the previous lemma implies that $\Phi_{r}\left(F\left(X_{c}\right)\right)=F_{r}\left(X_{c}\right)$. Moreover, parts 2 and 5 of that lemma imply that $g_{r}$ is a diffeomorphism of $D_{\infty} \backslash\left\{F\left(X_{c}\right)\right.$ : $|c| \leq 1 / 2\}$ to $D_{r} \backslash\left\{F_{r}\left(X_{c}\right):|c| \leq 1 / 2\right\}$. Since $\mu$ is constant on each $F\left(X_{c}\right)$, this implies that $\Phi_{r}$ is a diffeomorphism of $D_{\infty}$ to $D_{r}$. In fact, setting $\gamma(p)=\mu\left(F\left(F_{r}^{-1}(p)\right)\right)$, we have

$$
\Phi_{r}^{-1}(p)=g_{r / \gamma(p)}^{-1} F_{r / \gamma(p)} F_{r}^{-1}(p) .
$$

Moreover, if $p$ is in the neighborhood of $\partial V \cup \overline{\Delta_{0}^{2}}$ where $\mu=1$ and $g_{r}=I d$, then $\Phi_{r}(p)=p$. Similarly, if $p$ is in the neighborhood of $F(\partial V) \backslash F\left(\overline{\Delta_{0}^{2}}\right)$ where $\mu=0$, then $\Phi_{r}(p)=F_{r} F^{-1}(p)$. Finally, if $p \in F(\partial V) \cap F\left(\overline{\Delta_{0}^{2}}\right)$, then $F_{s}=F$ at $F^{-1}(p)$ for any $s \geq r_{0}$, and hence $g_{s}(p)=p$ and thus $\Phi_{r}(p)=p$. Thus $\Phi_{r}$ satisfies all of the needed requirements.

\section{Behavior on the sphere at infinity}

In this section we describe the behavior of the map $F_{r}$ on the 3 -sphere at infinity. Much of this section is similar to and inspired by [8], where the theory of solenoidal mappings is developed more fully.

Let $\mathbb{T}=D \times S^{1}$, where $D$ is the disk of radius 2 in $\mathbb{C}$, and let $(z, \zeta)$ be the coordinates in $\mathbb{T}$. Let $C_{+}$and $C_{-}$be the families of cones

$$
\begin{aligned}
& C_{+}(z, \zeta)=\left\{(u, \xi) \in T_{(z, \zeta)} \mathbb{T}:|\xi| \geq|u|\right\} \\
& C_{-}(z, \zeta)=\left\{(u, \xi) \in T_{(z, \zeta)} \mathbb{T}:|\xi| \leq|u|\right\}
\end{aligned}
$$

in the tangent bundle of $\mathbb{T}$. Following [8], we say that a map $\tau: \mathbb{T} \rightarrow \mathbb{T}$ is solenoidal of degree $d$ if $\tau$ is a $C^{1}$ injective immersion of degree $d$ such that $D \tau\left(C_{+}\right) \subseteq C_{+}$, and if there exists a constant $K>1$ such that for all $(z, \zeta) \in \mathbb{T}$ we have that $(u, \xi) \in C_{+}(z, \zeta)$ and $D_{(z, \zeta)} \tau(u, \xi)=\left(u_{1}, \xi_{1}\right)$ imply $\left|\xi_{1}\right|>K|\xi|$ and that $(u, \xi) \in C_{-}(z, \zeta)$ and $D_{(z, \zeta)} \tau(u, \xi)=$ $\left(u_{1}, \xi_{1}\right)$ imply $\left|u_{1}\right|<1 / K|u|$.

A standard model of a solenoidal mapping is given by defining

$$
\tau_{d}(z, \zeta)=\left(\zeta+\epsilon z \zeta^{1-d}, \zeta^{d}\right)
$$


for $(z, \zeta) \in \mathbb{C}^{2},|z| \leq 2,|\zeta|=1$. In [8] and [9], two different methods are given for extending a Hénon map to a compactification of $\mathbb{C}^{2}$ so that the compactification is homeomorphic to a closed 4-ball and so that the Hénon map extends to a homeomorphism of this closed 4-ball to itself. Moreover, for both methods, the authors show that on the sphere at infinity, this homeomorphism has an attracting solenoid and a repelling solenoid, and that in a neighborhood of the attracting solenoid the homeomorphism is conjugate to $\tau_{d}$, where $d$ is the degree of the Hénon map. In a neighborhood of the repelling solenoid, the homeomorphism is conjugate to $\tau_{d}^{-1}$.

These results are also true for the present extension. Let $\hat{H}_{r}$ denote the map induced by $H_{r}$ (or equivalently $F_{r}$ ) on the 3 -sphere. Let $V^{+}=\left\{(x, y):|x|<2|y|,|x|^{2}+|y|^{2}=1\right\}$. Note that $V^{+}$is a solid torus and that by lemma 3.1, $\hat{H}_{r}$ maps $V^{+}$into the interior of $V^{+}$. Also, let $\sigma(x, y)=(y, x)$.

THEOREM 4.1 For $r$ sufficiently large, $\hat{H}_{r} \mid V^{+}\left(\right.$resp. $\hat{H}_{r}^{-1} \mid \sigma\left(V^{+}\right)$) is solenoidal and conjugate to $\tau_{d}$ (resp. $\left.\tau_{d}^{-1}\right)$.

Proof: We can write $\hat{H}_{r}$ in coordinates $(z, \zeta),|z| \leq 2,|\zeta|=1$ by

$$
\hat{H}_{r}(z, \zeta)=\left(\frac{\zeta}{\left|R \zeta^{d}-z\right|}, \frac{R \zeta^{d}-z}{\left|R \zeta^{d}-z\right|}\right),
$$

where $R=R(r)$. Conjugating $\zeta$ by the map $\theta \mapsto e^{i \theta}$, which induces an isometry on the tangent bundle, we can write $\hat{H}_{r}$ as

$$
\begin{aligned}
\hat{H}_{r}(z, \theta) & =\left(\frac{e^{i \theta}}{\left|R e^{i \theta}-z\right|}, \operatorname{Im} \log \left(R e^{i d \theta}-z\right)\right) \\
& =\left(\frac{e^{i \theta}}{R}\left|1-\frac{z e^{-i \theta}}{R}\right|^{-1}, d \theta+\operatorname{Im} \log \left(1-\frac{z e^{-i d \theta}}{R}\right)\right) .
\end{aligned}
$$

From this, we see that as $r \rightarrow \infty$ (and hence $R \rightarrow \infty$ ), the partial derivatives of all terms except $d \theta$ tend to 0 . In particular, for $r$ sufficiently large, $\hat{H}_{r}$ satisfies the conditions for being solenoidal.

The fact that $\hat{H}_{r}: V^{+} \rightarrow V^{+}$is conjugate to $\tau_{d}$ follows as in corollary X.2 of [9]: $\hat{H}_{r}$ restricted to $V^{+}$is conjugate to one of the maps $\tau_{d, k}$ as in theorem 3.11 of [8], and proposition 4.6 of [8] implies that $\tau_{d, 0}=\tau_{d}$ is the only one of these maps which can be extended to an orientation preserving homeomorphism of the 3 -sphere. Since $\hat{H}_{r}$ extends, it must be conjugate to $\tau_{d}$. Since $\hat{H}_{r}^{-1}$ is conjugate to $\hat{H}_{r}$ by the involution $\sigma(x, y)=(y, x)$, we see that $\hat{H}_{r}^{-1}$ is conjugate to $\tau_{d}^{-1}$ on $\sigma\left(V^{+}\right)$.

In general, $\hat{H}_{r}$ need not be conjugate to $\hat{H}_{s}$ on all of the 3 -sphere for $r \neq s$. John Hubbard is planning a detailed study of the conjugacy classes of maps similar to $\hat{H}_{r}$.

\section{Smooth results}

In this section we modify the proofs of the previous sections to obtain the same results except that here the maps $F_{r}$ and the one parameter family are all $C^{\infty}$ smooth. 
Let $F, r_{0}, r$, and $R=R(r)$ be as before, and define

$$
J_{r}(x, y)=\left(y, R \frac{y^{d}}{|y|^{d-1}} \phi\left(\frac{|x|}{C R|y|}\right)-x\right)
$$

where $C=C(r)$ is a positive function to be specified. Then $J_{r}$ is $\mathbb{R}^{+}$homogeneous of degree 1 , and $J_{r}$ is $C^{\infty}$ on $\mathbb{C}^{2} \backslash\{(0,0)\}$.

PROPOSITION 5.1 There exists a one-parameter family of $C^{\infty}$ diffeomorphisms $F_{r}, r \geq$ $r_{0}$ of $\mathbb{C}^{2}$ and a $C^{\infty}$ function $R_{0}(r)>r$ such that $F_{r}(x, y)=F(x, y)$ if $\|(x, y)\|_{\infty}<r$ and $F_{r}(x, y)=J_{r}(x, y)$ if $\|(x, y)\|_{\infty}>R_{0}(r)$. Moreover, $F_{r}(x, y)$ is $C^{\infty}$ in $(r, x, y)$.

Proof: The proof is nearly the same as that for proposition 2.1. Referring to that proof, define $\alpha_{r}(y)$ as before, define $\beta_{r}=\sup \left\{\left|p_{r}(y)\right|+\left|S_{r}(y)\right|:|y| \leq 1\right\}$, then choose $L(r)$ a positive $C^{\infty}$ function so that $L(r) \geq \max \left\{\beta_{r}, \sup \left\{\alpha_{r}(y): y \in \mathbb{C}\right\}\right\} /|a|$.

Define $\psi_{r}$ as before with this new $L(r)$, let $M(r)=r+2+3 L(r)$, and let $C=C(r)$ be $C^{\infty}$ with $C(r) \geq \max \{24 /|a|, M(r), 2+1 /|a|\}$. Let

$$
q_{r}(x, y)=\psi_{r}(x) p_{r}(y)+\left(1-\psi_{r}(x)\right) S_{r}(y) \phi\left(\frac{|x|}{C R|y|}\right)
$$

and

$$
G_{r}(x, y)=\left(y, q_{r}(x, y)-a x\right)
$$

Since $\left|\phi^{\prime}\right| \leq 2$, we have for any $x_{1}, x_{2} \in \mathbb{C}$ that

$$
\begin{gathered}
\left|\frac{\partial}{\partial t} q_{r}\left(x_{1}+\left(x_{2}-x_{1}\right) t, y\right)\right| \leq\left|S_{r}(y)\right| \frac{2\left|x_{2}-x_{1}\right|}{C R|y|}\left(1+\psi_{r}\left(x_{1}+\left(x_{2}-x_{1}\right) t\right)\right) \\
\quad+\left|\frac{\partial}{\partial t} \psi_{r}\left(x_{1}+\left(x_{2}-x_{1}\right) t\right)\right|\left|p_{r}(y)-S_{r}(y) \phi\left(\frac{x_{1}+\left(x_{2}-x_{1}\right) t}{C R|y|}\right)\right| .
\end{gathered}
$$

Note that in this expression both $\psi_{r}$ and $(\partial / \partial t) \psi_{r}$ are 0 if $\left|x_{1}+\left(x_{2}-x_{1}\right) t\right| \geq M(r)$, in which case the right hand side is bounded by $|a|\left|x_{2}-x_{1}\right| / 12$. On the other hand, if $\mid x_{1}+\left(x_{2}-x_{1}\right) t \leq$ $M(r)$, then $\phi(|x| / C R|y|)=1$ whenever $|y| \geq 8 M(r) / C R$. Since $8 M(r) / C R \leq 1$, we see that if $\left|x_{1}+\left(x_{2}-x_{1}\right) t\right| \leq M(r)$, then

$$
\left|p_{r}(y)-S_{r}(y) \phi\left(\frac{x_{1}+\left(x_{2}-x_{1}\right) t}{C R|y|}\right)\right| \leq \max \left\{\beta_{r}, \alpha_{r}(y)\right\} \leq L(r)|a| .
$$

This plus equation (2.3) and $C \geq 24 /|a|$, implies that the right side of (5.1) is bounded by $5|a|\left|x_{1}-x_{2}\right| / 6$, so continuing as in the proof of proposition 2.1 , we see that $G_{r}$ is a diffeomorphism of $\mathbb{C}^{2}$.

Finally, with $a(r, x, y)$ as in the proof of proposition 2.1 , we can define

$$
F_{r}(x, y)= \begin{cases}G_{r}(x, y)=\left(y, q_{r}(x, y)-a x\right) & \text { if }\|(x, y)\|_{\infty} \leq M(r) \\ \left(y, S_{r}(y) \phi\left(\frac{|x|}{C(r) R(r)|y|}\right)-a(r, x, y)\right) & \text { if }\|(x, y)\|_{\infty} \geq M(r)\end{cases}
$$


Note that $\phi(t)=0$ for $t>1$, so $F_{r}$ is smooth even along $|y|=0$. Moreover, properties 4 and 6 of $a(r, x, y)$ together with the fact that

$$
\left|\frac{\partial}{\partial t} S_{r}(y) \phi\left(\frac{t}{C R|y|}\right)\right| \leq \frac{2}{C}
$$

and the fact that $C>2+1 /|a|$ implies that $F_{r}(\cdot, y)$ is a diffeomorphism of $\mathbb{C}$ for each fixed $y$, hence that $F_{r}$ is a diffeomorphism of $\mathbb{C}^{2}$. By property 3 of $a(r, x, y), F_{r}(x, y)=J_{r}(x, y)$ for $\|(x, y)\|_{\infty} \geq R_{0}(r)$, as desired.

THEOREM 5.2 The maps $F$ and $F_{r}$ are conjugate via a $C^{\infty}$ diffeomorphism of $\mathbb{C}^{2}$. I.e., there exists $\Phi_{r}: \mathbb{C}^{2} \rightarrow \mathbb{C}^{2}$ a diffeomorphism such that $\Phi_{r}^{-1} F \Phi_{r}=F_{r}$.

Proof: On the set $U$, the $F_{r}$ constructed in this section equals the $F_{r}$ constructed in proposition 2.1. Hence the conjugation in the current theorem is obtained exactly as in theorem 3.8.

The fact that $\Phi_{r}$ is $C^{\infty}$ follows from the facts that $\Phi_{r}$ is $C^{\infty}$ on $D_{\infty}$, that $F$ and $F_{r}$ are $C^{\infty}$, that $\Phi_{r}=I d$ on a neighborhood of $\partial V \cup \overline{\Delta_{0}^{2}}$ and $\Phi_{r}=F_{r} \circ F^{-1}$ on a neighborhood of $F(\partial V)$, and that $\Phi_{r}(p)=F_{r}^{-n} \Phi_{r} F^{n}(p)$ for $p \in F^{-n}\left(D_{\infty}\right)$.

Note that $J_{r}=H_{r}$ except at points $(x, y)$ where $|x| / C R|y| \geq 1 / 8$, which implies the equality of $\hat{J}_{r}$ and $\hat{H}_{r}$ except on the analogous set in the 3 -sphere. In particular, $\hat{J}_{r} \mid V^{+}=$ $\hat{H}_{r} \mid V^{+}$is still conjugate to $\tau_{d}$. Moreover, if $|x| / C R|y| \geq 1 / 8$ and $(z, w)=J_{r}(x, y)$, then $C \geq 24 /|a|$ implies that $|a x| / 2 \geq R|y|$, hence $|w| \geq|a x| / 2>2|z|$. Thus, for such $(x, y)$, the point on the 3-sphere corresponding to $J_{r}(x, y)$ is not contained in $\sigma\left(V^{+}\right)$. In particular, if $(z, w) \in \sigma\left(V^{+}\right)$, then $\hat{J}_{r}^{-1}(z, w)=\hat{H}_{r}^{-1}(z, w)$, so $\hat{J}_{r}^{-1}$ is conjugate to $\tau_{d}^{-1}$ on $\sigma\left(V^{+}\right)$by theorem 4.1.

\section{Compositions of Hénon maps}

In this section we obtain results similar to those in earlier sections, but here the focus is on compositions of Hénon maps.

Let $F_{j}(x, y)=\left(y, p_{j}(y)-a_{j} x\right)$ for $j=1, \ldots, n$, where $p_{j}$ is a polynomial of degree $d_{j} \geq 2$ having the form of the polynomial $p$ of section 2 and $a_{j} \geq 0$. As noted earlier, [6] implies that any polynomial automorphism of $\mathbb{C}^{2}$ with nontrivial dynamics is conjugate to the map $F=F_{n} \circ \cdots \circ F_{1}$ for some choice of $n$, the $p_{j}$ 's and the $a_{j}$ 's.

For each $j$, we can construct $F_{j, r}$ as in proposition 2.1 (or proposition 5.1). Let $r_{0}$ be the maximum of the $r_{0}$ 's corresponding to each individual $F_{j, r}$. Let $\hat{r}_{0}>0$ be large enough that $F\left(\overline{\Delta_{0}^{2}\left(0 ; \hat{r}_{0}\right.}\right)$ contains $\Delta^{2}\left(0 ; r_{0}\right)$.

THEOREM 6.1 Let $\mathbf{r}=\left(r_{1}, \ldots, r_{n}\right)$ with each $r_{j} \geq \hat{r}_{0}$. Then $F=F_{n} \circ \cdots \circ F_{1}$ is conjugate on $\mathbb{C}^{2}$ to $F_{\mathbf{r}}=F_{n, r_{n}} \circ \cdots \circ F_{1, r_{1}}$. If each $F_{j, r_{j}}$ is constructed as in proposition 5.1 , then the conjugating map is smooth. 
Proof: The proof of lemma 3.6 implies that the inequality in (3.1) is valid for each $F_{j, r}$ for all $r \geq r_{0},|x|<|y|,|y| \geq r_{0} / 2,|c|<1$, and $\theta$ real. By lemma 3.1, we can apply each $F_{j, r_{j}}$ in turn to see that $(3.1)$ is valid for $F_{\mathbf{r}}$. Hence lemma 3.6 is valid for $F_{\mathbf{r}}$.

For each $r \geq 0$, let $F_{\mathbf{r}, r}=F_{n, r_{n}+r} \circ \cdots \circ F_{1, r_{1}+r}$, let $F_{\mathbf{r}, \infty}=F$, and for $r \in[0, \infty]$, let $D_{r}=\left(F_{\mathbf{r}, r}(\bar{V}) \backslash V\right) \cap \overline{\Delta_{0}^{2}}$.

Since lemma 3.6 is valid in the current setting and by the choice of $\hat{r}_{0}$, we see that lemma 3.7 is valid with $F_{\mathbf{r}, r}$ in place of $F_{r}$ using the same proof (up to some notational changes). Hence the proof of theorem 3.8 is also valid in the current setting. Thus $F_{\mathbf{r}}$ and $F$ are conjugate as claimed, and the smoothness follows as in theorem 5.2.

Finally, for $r_{j}$ sufficiently large, the map induced on the 3 -sphere at infinity by $F_{j, r_{j}}$ will be solenoidal on $V^{+}$as in theorem 4.1, so the composition of these maps, $\hat{F}_{\mathbf{r}}$ will also be solenoidal on $V^{+}$, as can be seen by checking the definition of solenoidal. Moreover, $\hat{F}_{\mathbf{r}}$ is conjugate to $\tau_{d_{n}} \circ \cdots \circ \tau_{d_{1}}$ on $V^{+}$. This can be seen by applying proposition 3.8 of [8] inductively to obtain the conjugacy on a fundamental domain for $\hat{F}_{\mathbf{r}}$, then completing the conjugacy as in theorem 3.11 of [8]. Analogous results obtain for the inverses of the relevant maps. For further results on such compositions, the reader is urged to consult section XI of $[9]$.

\section{References}

[1] E. Bedford and J. Smillie, Polynomial diffeomorphisms of $\mathbb{C}^{2}$ : currents, equilibrium measure and hyperbolicity, Invent. Math., 103 (1991), no. 1, 69-99.

[2] E. Bedford and J. Smillie, Polynomial diffeomorphisms of $\mathbb{C}^{2}$. II. Stable manifolds and recurrence, J. Amer. Math. Soc., 4 (1991), no. 4, 657-679.

[3] G. Buzzard, Infinitely many periodic attractors for holomorphic maps of 2 variables, Ann. of Math., 145 (1997), 389-417.

[4] G. Buzzard, Kupka-Smale theorem for automorphisms of $\mathbb{C}^{n}$, Duke Math. J., 93 (1998), No. 3, 487-503.

[5] G. Buzzard and J. Smillie, Complex dynamics in several variables, Flavors of Geometry, MSRI Publications, Vol 31, Cambridge University Press, 1997.

[6] S. Friedland and J. Milnor, Dynamical properties of polynomial automorphisms, Ergod. Th. Dyn. Sys., 9 (1989), 67-99.

[7] M.W. Hirsch, Differential Topology, Graduate Texts in Math., 33. Springer-Verlag: New York, 1976.

[8] J.H. Hubbard and R. Oberste-Vorth, Hénon mappings in the complex domain, Publ. Math. I.H.E.S., 79 (1994), 5-46.

[9] J.H. Hubbard, P. Papadopol, and V. Veselov, to appear in Acta Math. 
[10] C. Robinson, Dynamical systems : stability, symbolic dynamics, and chaos, CRC Press: Boca Raton, 1995. 